\title{
Climbing and Turning Control of a Biped Passive Walker by Periodic Input Based on Frequency Entrainment
}

\author{
Soichiro Suzuki ${ }^{1, a}$, Ying Cao ${ }^{2, \text { b }}$, Masamichi Takada ${ }^{2}$ and Kentaro $\mathrm{Oi}^{2}$ \\ ${ }^{1}$ Dept. of Mechanical Engineering, Kitami Institute of Technology, Japan \\ ${ }^{2}$ Graduate School of Kitami Institute of Technology, Mechanical System Engineering, Japan \\ azuki@mail.kitami-it.ac.jp, bcaoying_0112@yahoo.co.jp
}

Keywords: passive walker, climbing control, turning control, periodic input

\begin{abstract}
This study is aimed at stabilizing a three dimensional biped passive walker in various environments and achieving climbing and turning control. The novel control method synchronizes a period of the changing motion of the stance leg in frontal plane (frontal motion) with a period of the swing leg by periodic input in order to stabilize the three dimensional passive walker. A mechanical oscillator is utilized to change the period of the frontal motion. The target path of the oscillator is automatically generated based on frequency entrainment in order to adjust the period of the frontal motion. In the climbing and turning control of the passive walker, the amplitude and the phase generating algorithm of the target path of the oscillator are improved. It is analytically demonstrated that the biped passive walker can be stabilized even in climbing and turning.
\end{abstract}

\section{Introduction}

Researches of biped robots are always fascinating many people because they hope that the biped robots can substitute for human in various environments including dangerous, tiresome or repetitive situations. However, the problems on stability in random environment and high energy consumption have not been solved. At present most bipedal robotic walk is based on precise joint-angle control, which requires higher precision and frequency response of actuators than human muscles [1]. This means that the energy consumption of robots is so high. To address this issue, passive dynamic walkers were proposed with a new design and control paradigm [2]. Passive walkers can walk down a slope by its mechanical structure and gravitational potential energy with startling human-like gaits [1, 3]. However, it is difficult to stabilize passive walkers in uneven terrain, such as changeable slopes. Presently it is demonstrated that there are some kinds of stability criteria and stabilizing method for passive walkers. McGeer and Goswami analyzed the stability of passive walkers by Poincare return map $[2,4]$, but it could not be applied to non-periodic walking, such as turn or walking on changeable slopes. Tedrake developed a quasi-passive walker with learning ability by using a reinforcement learning method [5]. Wisse used skate board-like ankle joints to provide 3D stability. These ankles couple the unstable sideways lean motion to yaw (steering) [6].

In this study, it was experimentally demonstrated that synchronization of the changing motion of the stance leg in the frontal plane (frontal motion) with the swing leg motion became necessary condition for stable passive walk, and a passive walker was stabilized on various slope angles by regulating some mechanical parameters of it [7]. In the next step, a mechanical oscillator was set on the passive walker, which always synchronized the period of the frontal motion with the period of the swing leg [8].

The experimental quasi-passive walker with a mechanical oscillator is shown as Fig.1. A pulse motor and a mechanical oscillator are fixed on the top of the body. An acceleration sensor is also fixed on the body to measure the rocking motion angle $\theta$ in frontal plane, and the swing leg angle is measured by a rotary encoder. The dynamic model of the frontal motion can be simplified as shown in Fig.2. By solving the Lagrange equations of the dynamic model, it was demonstrated that the period of the frontal motion could be changed by the torque of the oscillator. Therefore, it is inferred that the period of the frontal motion can be controlled indirectly by controlling the period, the amplitude and the phase of the oscillator. 


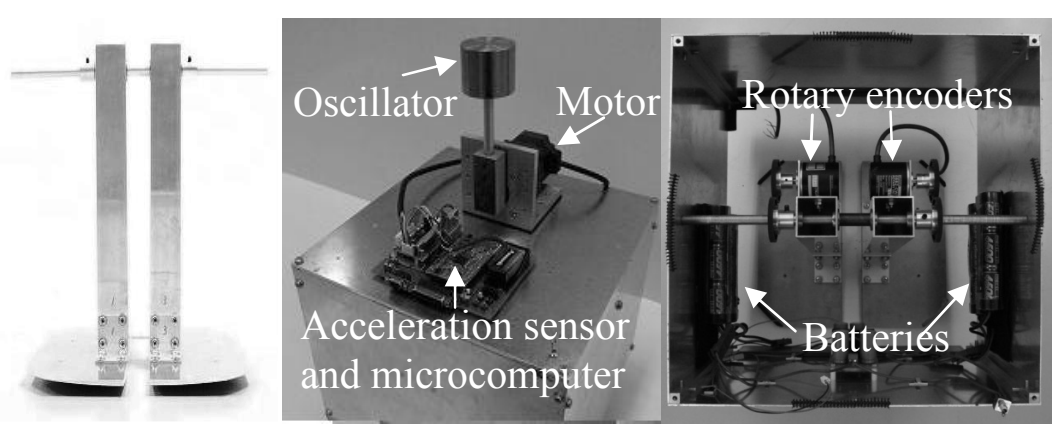

Fig. 1. A passive walker and a mechanical oscillator

The original control algorithm is shown briefly as follows. In order to synchronize the frontal motion with the swing leg motion, the target path of the oscillator is automatically generated to control the period of the frontal motion by controlling the period, the amplitude and the phase of the oscillator, respectively [8]. When the rocking angle $\theta$ of the frontal motion is input into van der Pol equation, the period of the target path of the oscillator can be calculated by numerical solution of the equation. According to frequency entrainment of Van der Pol equation, the period of the target path becomes equal to the period of the frontal motion. The amplitude of the target path of the oscillator is proportionally determined by periodic input, the difference of the period of the frontal motion and the swing leg motion. The larger the amplitude is, the larger the change of the period of the frontal motion is. The phase difference $\varphi$ between the target path of the oscillator and the frontal motion is set to be $90[\mathrm{deg}]$ or $-90[\mathrm{deg}]$ when the period of the frontal motion need to be increased or decreased, respectively. This method can change the period of the frontal motion most efficiently from a point of view of energy transform.

The goal of this research is to stabilize the three dimensional biped passive walker in various environments and achieving climbing and turning control. A new control method that is able to stabilize the passive walker not only in downward slope but also horizontal floor and upward slope including climbing and turning by using a mechanical oscillator is examined. Firstly, construction of the passive walker is improved in order to enhance the mechanical property. Secondly, novel climbing and turning control algorithms are examined. Finally, effectiveness of the algorithms utilizing the frequency entrainment is demonstrated in numerical simulation.

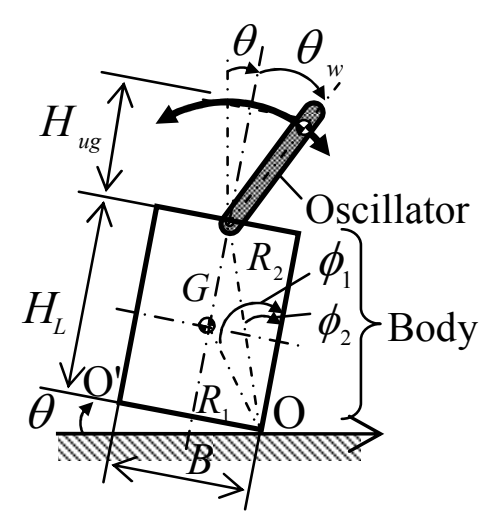

Fig. 2. A dynamic model of the frontal motion

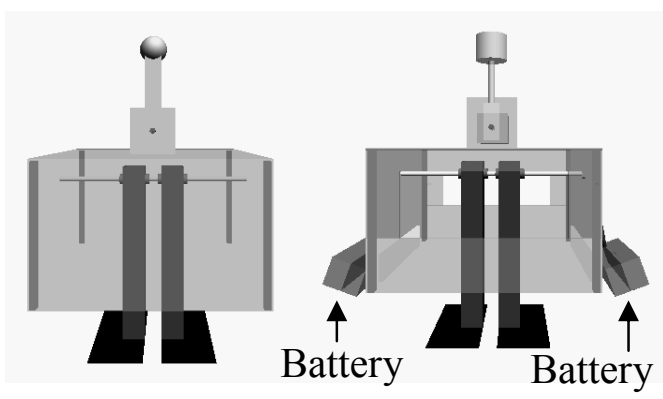

Fig. 3. Existent and improved models

\section{Improvement of mechanical construction}

In order to examine the climbing and turning control methods, it is necessary to enhance the stability on the mechanical properties of the passive walker. Existent and improved simulation models are shown in Fig.3.

Firstly, the moment of inertia of the passive walker around a yaw axis of foot pivot is increased. The existent model (a left picture of Fig.3) easily rotates around the yaw axis, because its spherical foot bottom touches the ground on one pivot. Since the rotating motion makes the walk unstable, it is 
necessary to decrease the rotating motion. To solve this problem, batteries are placed as counterbalance on the arms both sides of the body to increase the moment of inertia as shown in a right picture of Fig.3. In addition, the center of mass of a leg is regulated backward on the foot so that the legs can naturally swing forward even on an upward slope in swing phase.

\section{Climbing control algorithm and numerical simulation}

3.1 Climbing Control algorithm. In order to perform climbing control, the algorithm for determination of the amplitude $\beta$ of the target path of the mechanical oscillator is improved as shown in Fig.4. From the difference between swing leg periods $\mathrm{T}_{\mathrm{S}}$ and $\mathrm{T}_{\mathrm{S}-1}$, which is defined as $\mathrm{T}_{\text {dif }}$, the change of inclination of ground can be inferred. Therefore, the amplitude $\beta$ is automatically generated based on three different conditions of $\mathrm{T}_{\text {dif }}$ to keep stable frontal motion of the passive walker. The period of the oscillator is generated by van der Pol equation utilizing the frequency entrainment as same as the original control algorithm.

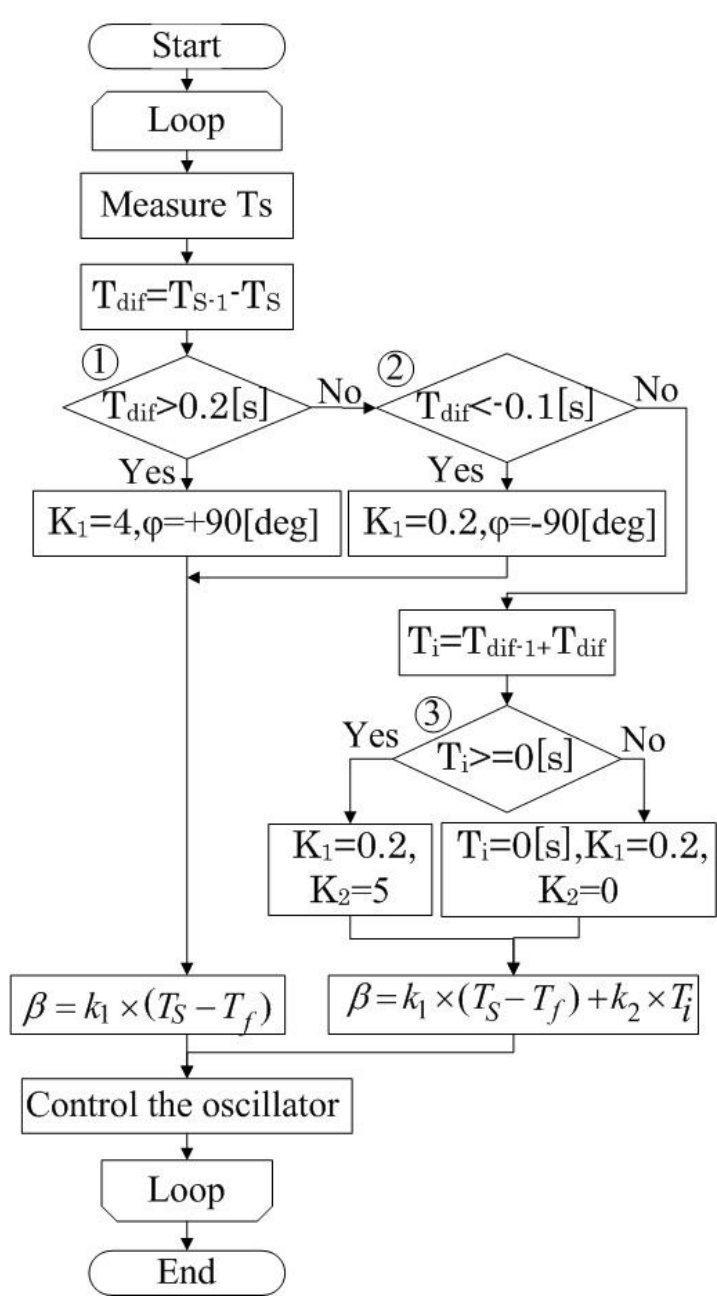

Fig. 4. Climbing control algorithm

At distinction (1), if the period of the swing leg largely decreases, $\mathrm{T}_{\text {dif }}$ is larger than $0.2[\mathrm{~s}]$. It can also be inferred that the inclination of ground increases. In this condition, in order to stabilize the gait of the passive walker in climbing, the phase difference $\varphi$ is set to be $+90[\mathrm{deg}]$ so that the rocking angle $\theta$ can increase. The amplitude $\beta$ of the oscillator is determined as the product of the proportion gain $\mathrm{K}_{1}$ which is set at 4 and the difference between $T_{\mathrm{s}}$ and the period of the frontal motion $\mathrm{T}_{\mathrm{f}}$.

At distinction (2), if the period of the swing leg increases because of a down slope or some interference, $\mathrm{T}_{\text {dif }}$ is smaller than $-0.1[\mathrm{~s}]$. In this condition, the rocking angle $\theta$ should be reduced, so the phase difference $\varphi$ is set to be $-90[\mathrm{deg}]$, and the proportion gain $\mathrm{K}_{1}$ is set at 0.2 . In the other condition of the distinction (2), $\mathrm{T}_{\mathrm{dif}}$ is between $-0.1[\mathrm{~s}]$ and $0.2[\mathrm{~s}]$. This means that the period of the swing leg slightly changes and the walking is stable. In the original algorithm, because the target amplitude is determined by the difference $T_{s}-T_{f}$, the amplitude becomes too small. Therefore, in the new control algorithm, $\mathrm{T}_{\mathrm{i}}$, which is defined as a summation of $\mathrm{T}_{\text {dif }}$ and the last period difference $\mathrm{T}_{\text {dif-1 }}$, is used to estimate whether the period of the swing leg is increasing or decreasing.

At distinction (3), if the period of the swing leg decreases during last two steps, $\mathrm{T}_{\mathrm{i}}$ is larger than 0 or equal to 0 . To keep the period of the swing leg long enough, $\mathrm{T}_{\mathrm{i}}$ is used to increase the amplitude of oscillator as shown in Eq. 1. In this case, the phase difference $\varphi$ is determined by the amplitude $\beta$. If the amplitude $\beta$ is positive, $\varphi$ is set to be 90 [deg], and is negative, $\varphi$ is $-90[\mathrm{deg}]$.

$$
\beta=k_{1} \times\left(T_{S}-T_{f}\right)+k_{2} \times T_{i} .
$$

3.2 Numerical simulation. Based on the algorithm mentioned above, the climbing control is analytically examined by using Open Dynamic Engine (ODE). The walking course is continuously set as a flat ground and an upward slope of $3[\mathrm{deg}]$ inclination. The numerical simulation results of change of the period of the swing leg motion $\mathrm{T}_{\mathrm{S}}$ and the period of the frontal motion $\mathrm{T}_{\mathrm{f}}$ are shown in 
Fig.5. When the inclination of the slope increases, the period of the swing leg $\mathrm{T}_{\mathrm{S}}$ sharply decreases. However, the proportional gain for the determination of the amplitude is changed according to the climbing control algorithm in order to increase $T_{S}$ and $T_{f}$. As a result, the passive walker is stabilized by synchronizing $\mathrm{T}_{\mathrm{f}}$ with $\mathrm{T}_{\mathrm{S}}$.

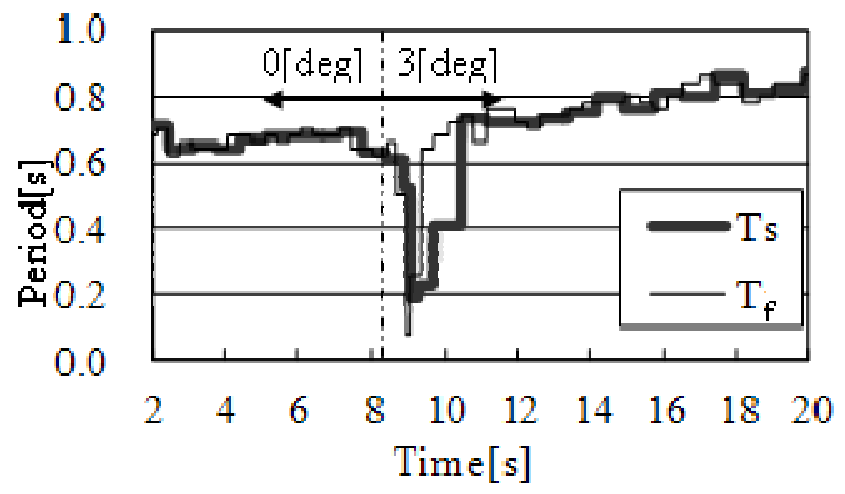

Fig. 5. Simulation results of change of $T_{S}$ and $T_{f}$

\section{Turning control algorithm and numerical simulation}

4.1 Turning Control Algorithm. In this research, the turning control utilizes the difference between the right and left strides. For example, if the right stride is shorter than the left stride, the passive walker turns right. In order to change the strides, the phase of the oscillator is regulated. Therefore, the oscillator separately has two target paths for changing both strides. In the original control algorithm the phase difference of the oscillator and the frontal motion $\varphi$ is always set to be 90 [deg] or $-90[\mathrm{deg}]$ to increase or decrease the period of the frontal motion, respectively. In the turning control algorithm, if $\varphi$ of the target path of the oscillator on one side is set as negative at the single support phase in the walking cycle, $\varphi$ of the target path for the opposite side should be set as positive so that the period of the swing leg of the opposite side is shortened. As a result, the passive walker turns to this side. On the other hand, the period of the oscillator is automatically generated by van der Pol equation based on the frequency entrainment as same as the original control algorithm.

4.2 Numerical simulation. Effectiveness of the turning control algorithm mentioned above was examined by a numerical simulation. The left turning route on a flat ground is changed by regulating the phase difference $\varphi$ between the oscillator and the frontal motion as shown in Fig.6. Phase differences $\varphi$ on both sides are set from \pm 90 [deg] to \pm 170 [deg], respectively. The amplitude of the target path of the oscillator $\beta$ is always fixed at $11[\mathrm{deg}]$.

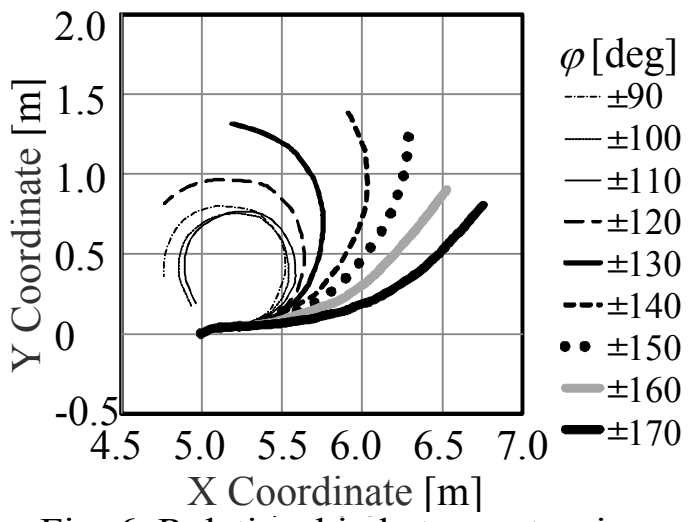

Fig. 6. Relationship between turning radius and phase difference

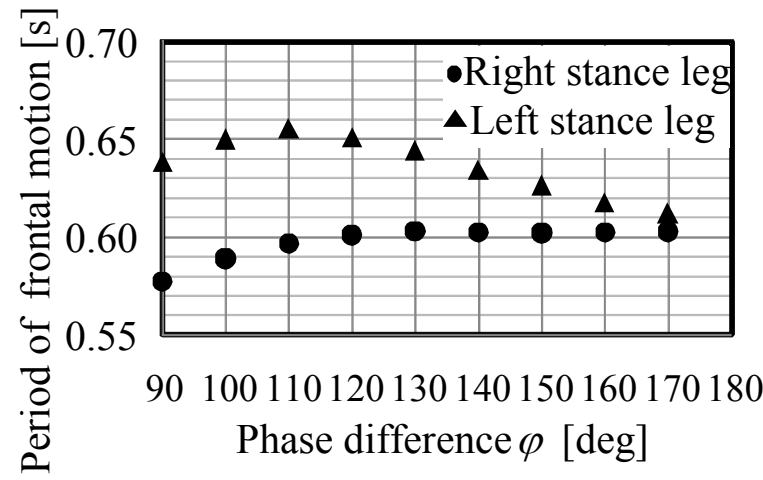

Fig. 7. Relationship between period of the frontal motion and phase difference 
As shown in Fig.6, the turning route could be changed by the setting of $\varphi$, and basically, the larger the absolute value of $\varphi$ is, the larger the turning radius is. In the previous study, it was demonstrated that $\varphi$ set at \pm 90 [deg] can most efficiently change the kinetic energy of the frontal motion. Therefore, it is expected that the small absolute value of $\varphi$ makes the turning radius small. However, when $\varphi$ is set at \pm 110 [deg], the turning radius is the smallest. The reason can be found from the change of periods of left and right stance legs with different $\varphi$ in Fig.7. When $\varphi$ is \pm 90 [deg], the difference of periods, between left and right stance legs, is almost the same as the difference of periods when $\varphi$ is \pm 110 [deg]. However, when $\varphi$ is \pm 110 [deg], the period of the stance leg is the longest, which makes the passive walker turn with larger strides. On the other hand, when $\varphi$ is \pm 90 [deg], discontinuousness of the target paths between both sides at switching becomes largest, so efficiency of energy transmission between the oscillator and the passive walker will be low. Therefore, it is expected that the turning radius does not become the smallest when $\varphi$ is \pm 90 [deg].

\section{Conclusion}

In this paper, the climbing and turning control algorithms of a three dimensional biped passive walker by periodic input based on the frequency entrainment were examined. It was analytically demonstrated that the biped passive walker was stabilized when the frontal motion period was synchronized with the swing leg period even in climbing and turning.

The mechanical construction of the passive walker was improved by increasing the moment of inertia around a yaw axis of the passive walker to enhance the stability on the mechanical properties in climbing and turning.

In the climbing control, the change of the period of the swing leg was used to estimate the walking statement and distinguish the control algorithm. However, the judgment criteria of walking statement mostly rely on results of simulations or experiments. In further research, the climbing control algorithm will be improved on this point.

In the turning control, the phase of the oscillator was regulated to control the turning route of the passive walker. The relationship between the turning radius and the phase difference $\varphi$ was demonstrated by the numerical simulation. In further research, the stability of turning will be improved in more complicated environments.

\section{References}

[1] S. Collins, et al, Efficient bipedal robots based on passive-dynamic walkers, J. Science. 307(5012) (2005) 1082-1085.

[2] T. McGeer, Passive dynamic walking, J. International Journal of Robotics Research. 9(2) (1990) 62-82.

[3] S. H. Collins, M. Wisse, A. Runia, A three-dimensional passive-dynamic walking robot with two legs and knees, J. The International Journal of Robotics Research. 20(7) (2001) 607-615.

[4] Goswami A, Espiau B, Keramane A, Limit cycle and their stability in a passive biped gait, Proc. of the IEEE International Conference on Robotics and Automation. Minneapolis, USA, (1996) 246-251.

[5] Tedrake R, Zhang T W, et al, Actuating a simple 3D passive dynamic walker, Proceedings of IEEE International Conference on Robotics and Automation. (2004) 4656-4661.

[6] Wisse M, Three additions to passive dynamic walking; actuation, an upper body, and 3D stability, Proceedings of IEEE-RAS International Conference on Humanoid Robots. (2004) 113-132.

[7] S. Suzuki, M. Hachiya, Experimental Study on Stabilization of a Three-Dimensional Biped Passive Walking Robot, Journal of the Society of Biomechanisms. 32(4) (2008) 239-246.

[8] M. Hachiya, S. Suzuki, Stabilization of a Biped Quasi Passive Walking Robot via Periodic Input, Journal of the Society of Biomechanisms. 33(1) (2009) 57-63. 